\title{
On Some Issues of Conceptualization and Categorization by Means of Natural Language
}

\author{
Victoria Dobrova ${ }^{l, *}$, Alexander Zhuravlev ${ }^{l}$, Irina Sysueva ${ }^{l}$, Svetlana Menshenina ${ }^{l}$, and Elchin Gashimov ${ }^{2}$ \\ ${ }^{1}$ Samara State Technical University, Department of Foreign Languages, 244 Molodogvardeyskaya Str., Samara 443100, Russia \\ ${ }^{2}$ Samara Branch of Moscow State Pedagogic University, Department of English Philology, 76 Stara Zagora Str., Samara, Russia
}

\begin{abstract}
This paper deals with a number of problems that occur most frequently in the course of scientific research during the process of conceptualization and categorization of the world around us by means of natural language. The main subject is to consider the origins of certain issues and difficulties concerning the process of conceptualization and categorization, as well as to explain their nature or, at least, work out some speculations. The methodology of the research is analysis of the most common problems that occur in the process of conceptualization and categorization of the world by means of natural language. Results of the research are determination of a number of problems concerning conceptualization and categorization, explanation of certain reasons for them to occur. A scope of results is various kinds of scientific research works, concerning the features and issues occurring in the course of conceptualization and categorization during the process of creating and analyzing different linguistic world images.
\end{abstract}

\section{Introduction}

History of origin and development of cognitive linguistics as a separate scientific area is an ocular demonstration of the fact that such notions as 'cognition', 'concept' and 'category' are an integral part of any language. In other words, one cannot speak about conceptualization and categorization as processes of perception and description of the world performed by a human without mentioning the role that natural language plays in these processes, i.e. about a cognitive function of language.

Today cognitive linguistics is in line with other cognitive sciences but it took it quite a long time to become an independent scientific area. It was recognition of the role that language played in cognitive activity that brought cognitive linguistics to life.

This is what E. Kubryakova thinks about the process of establishment of cognitive linguistics as the independent field of science, "We believe that the true revolution in linguistics was the shift from a general approach to a cognitive approach, the latter being aimed at new problems of analysis of knowledge structures and their connection with language and, consequently, with the research of categorization and conceptualization of the world. As the matter of fact it marked the beginning of cognitive linguistics as an independent theoretical discipline and consolidated its status as one of the leading areas of knowledge in cognitive sciences" [1].

V. Demiyankov calls it "linguistic turn of mind": "Linguistic turn, or, more precisely, mind being turned to language, means increased attention to language, to the way the (deep) structure of language is represented in the discourse of liberal arts - philosophy, literature, history, social science $<\ldots>$ previously one spoke about the subject (especially about a human as a research subject); now one speaks about natural language as an organizing link of scientific discourse which concerns existence, reality and human's thinking" [2].

$\mathrm{V}$. Maslova also noted the significance of shifting from "looking inwards" to "looking outwards": "The most important achievement of modern linguistics is that language is no longer treated as "something on its own and for its own"; in terms of new paradigm language is considered to be the part of human's cognitive activity" [3].

E. Golovanova is more straight about this point: "One distinctive feature of the cognitive approach as a new scientific paradigm is recognition of special role of linguistics which is caused by new evaluation of the role that language plays in human's verbal and cogitative activity. In cognitive scientists' opinion, language provides a better access to many processes of cogitative, cognitive nature and processes of conceptualization and categorization that cannot be observed directly" [4].

E. Golovanova gives a more precise definition of the role that language plays in cognitive activity: "The cognitive approach to study of language phenomena is based on the concept that the basis of language as a semiotic system and a special kind of activity is conceptual world image which is formed in human's mind as a result of his cognitive activity and during the process of communication and transforming activity.

*Corresponding author: victoria_dob@mail.ru 
Language itself serves as a cognitive mechanism which directly participates in forming of this system" [4].

In order to sum up what has been said during the discussion of the role of language in cognitive activity, we quote V. Maslova: "Cognitive science focuses on the ways and methods of processing, storage and translation of knowledge by the human $\langle\ldots\rangle$ Cognitive activity results in the system of meanings that belong to what a person knows and thinks about the world around him". V. Maslova also noted the connection between cognitive processes and language and concludes: "Understanding of that led to separation of cognitive science from cognitive linguistics" [3].

\section{Conceptualization Issues}

A concept is one of the most important notions of cognitive linguistics (along with a category and cognition) [4]. The notion of the concept is used by the number of liberal arts, e.g. in philosophy, psychology and cultural studies. Both the concept as a unit of knowledge and a process of conceptualization play an enormous role in cognition of the world and making up its linguistic image. V. Maslova, the author of the 'Cognitive Linguistics' study guide, says: "All cognitive activity (cognition) of a human can be considered as developing ability of finding one's way around the world, and this activity involves the necessity of identifying and distinguishing objects: concepts occur to supply this kind of operation. Consequently, conceptualization is connected with cognition of the world" [3].

N. Boldyrev also notes the role that conceptualization plays in human's cognition of the world: “... any type of knowledge that a human obtains is the result of conceptualization and categorization of the world around him. Consequently, language knowledge which is the integral part of human's general conceptual system is formed according to the same principles" [5].

Pretty much the same idea can be found in one of the scientific papers by A. Sharandin: "As everyone knows, conceptualization is connected with comprehension of incoming information, primarily about the world around, i.e. it is connected with the process of mental construction of its subjects and phenomena which leads to representing certain bits of knowledge about the world in form of concepts. In these terms the notion of conceptualization reflects continuous nature of perception and comprehension of the world, as well as its static result in form of certain mental units" [6].

E. Golovanova also touches upon the idea of 'mental construction': "Basic elements of the conceptual world image are concepts - meaningful operative units of knowledge. These very units and structures are used by human in the process of thinking and verbal and cogitative activity" [4].

The following line can be considered as some kind of summing up the discussion about how important is the concept for the process of analysis of incoming information, as well as for the process of synthesis of some higher-level structures on the basis of these concepts: "At the present moment we should admit that nothing else but the concept is the key notion of cognitive linguistics" [7].

Perhaps, one of the main issues of conceptualization by means of natural language is that the researchers are not uniform about a definition of concept $-\mathrm{a}$ definition which would describe its nature, characteristics and functions most precisely. This issue was particularly mentioned by Z. Popova and I. Sternin: “... in spite of the fact that the notion of concept may be considered firmly established in modern cognitive science, the meaning of this notion varies quite significantly depending on different conceptions of various scholarly traditions and single scientists" [7].

However, despite all difference between existing definitions of the 'concept' notion, many cognitive scientists agree that concept is a mental unit. That is what E. Golovanova thinks about it: "Concept belongs to mental level, consciousness level" [4]. Z. Popova and I. Sternin express the similar idea: "Concept belongs to human consciousness; it is the global unit of mental activity" [7].

These researchers also give a more comprehensive definition of concept: "We define concept as a discrete mental entity which is the basic unit of a human's cogitative code and which has regularized inner structure; it is the result of cognitive activity of a person and society, it holds comprehensive, encyclopedic information about the object or phenomenon it denotes, about interpretation of this information by social conscience and the way social conscience treats this object or phenomena" [7].

At last, let us consider how the acknowledged Russian expert in the field of cognitive science E. Kubryakova defines the concept: "A concept is an operative unit of the memory, mental vocabulary, conceptual system and brain language, the whole image of the world; it is a quantum of knowledge. The most important concepts have lexical representation" [8].

As we proceed on discussing some issues concerning the process of conceptualization of the world, we touch upon the question of connection between language and brainwork. To be more precise, we are going to talk about the connection between language and cognitive activity, or, in other words, about the role that natural language plays in the process of conceptualization and some related problems.

Basically, human's mental activity is not verbal by its nature. The passage from 'Cognitive linguistics' by $\mathrm{Z}$. Popova and I. Sternin runs as follows: "Human brainwork is non-verbal, it operates by means of universal subject code. People think with concepts that are coded by the bits of this code and these concepts form the basis of universal subject code" [7].

Consequently, concepts that are formed in the process of cognitive activity are also non-verbal in the first place. Concepts are objectified by means of natural language but it does not happen in 100 per cent of cases. There is no direct relationship between a concept and its linguistic representation. There are even two opposite points of view on the question "Do we really need a language to think with concepts?" among the cognitive 
scientists. Some of them - so-called 'verbalists' maintain that brainwork requires language as a tool for representation of knowledge to pass it along as information. Among these scientists are M. Müller, W. von Humboldt, F. Schleiermacher, F. de Saussure, A. Reformatskiy and others [9]. Other researchers (such as N. Zhinkin, J. Piaget, B. Serebrennikov and P. Galperin [9]) believe that language is not an obligatory attribute of cognitive thinking as a human thinks on pre-linguistic level and operates by non-verbal concepts in the first place.

In this study, we as researchers find it irrelevant to consider solely one of these approaches. We think that in our case it is more appropriate to talk about some kind of diachrony, about gradual transition from one approach to another. We can easily illustrate what we mean by the following example. Imagine there is a child; the process of his mental and linguistic development somehow reflects that of the mankind in general.

So, at the first stage of development a child's brainwork in non-verbal for he obviously does not speak any language. But his brain is already forming first nonverbal concepts. At this phase, the child's brain utilizes the pre-linguistic system of images - a primitive version of what is to become the universal subject code (USC). There is another passage from the book by Z. Popova and I. Sternin: "Mental activity doesn't always mean reference to language. Brain operates by means of universal subject code" [7]. As the child grows older he learns and interacts with other people thus growing to the certain level of consciousness, and he comes to using a language to objectify the concepts by words. E. Kubryakova says, "No generalization of human experience is possible out of a language, without a language" [1].

Summing up what has been said above we may say that a human can certainly perform mental and cognitive activities without direct reference to language. But, at the same time, we must admit that a person not speaking any language would look at least strange and not natural in modern society.

Another issue concerning the process of conceptualization of the world is connection between mental and linguistic units. In other words, we're going to speak how one and the same concept may be objectified by different people.

While we realize the important role that natural language plays in the process of conceptualization, we should also keep in mind a simple idea: language is just means of representation and objectifying of concepts: "The cognitive approach to study of linguistic phenomena is based on the understanding of the fact that language as a semiotic system and a form of activity is based on the system of knowledge about the world, i.e. the conceptual world image which is formed in human's mind as a result of his cognitive activity and in the process of communication and transformation activity. Language itself is considered to be one of cognitive mechanisms that form this system." [4].

Z. Popova and I. Sterning are more straight and precise about this idea: "Language $<\ldots>$ is just one of the methods of forming concepts in human's mind" [7].
E. Kubryakova believes that it is necessary to step away from "the idea that language reflects the world and that reality which ontologically exists out of us can be, so to speak, represented in language like a reflection in the mirror" [1].

Indeed, language is nothing more than an instrument, a means of actualization of non-verbal conceptual units. And, in our opinion, this instrument is quite imprecise or even rough and awkward. E. Golovanova says, "The main issue posed by cognitive approach in linguistics is the question of correlation between mental and linguistic units and structures" [4].

What caused this issue to arise? Why one and the same reality (and, as a result, completed conceptual world image) is represented differently in different people's minds and speech?

In one hand, all people think generally according to the same patterns, no matter what country they live in or what language they speak. N. Boldyrev notes: "Despite the fact that each person possesses unique knowledge about the world, basic cognitive processes of conceptualization (overthinking and consolidating the results of cognitive activity in form of knowledge units, i.e. concepts) and categorization (putting concepts into certain experience cells, i.e. categories) prove to follow the same patterns" [10]. E. Golovanova provides a more generalized comment: "Each consciousness is unique but all people's minds consist of universal components derived from people's common physiological and social needs" [4].

But anyway, all people think a little bit differently, that is why they have slightly different individual conceptual world images in their minds and that is why they use different ways to objectify the same concepts. This is what E. Kubryakova says about it: "The world is divided into concepts by a human and represented in language differently because in every natural language it is exclusively the result of processes of conceptualization and categorization that are performed slightly differently in different languages" [1]. E. Kubryakova goes on: "Languages of the world are examples of the fact that the world is divided into concepts on different basis, and this basis is defined by the specific features of languages, their origin and development in non-identical historical, geographical, ecological, sociological and cultural circumstances" [1].

O. Koval also underlines the influence of cultural characteristics on language: "Language reflects differences in mentality and culture between different nations" [11].

An example may be the number of different shades of green in language of Australian natives - this number is incommensurably bigger than in any European language. Linguistic and cultural prerequisites for that are more than obvious - tribes live in the jungle where green is dominant color. Consequently, conditions and processes of dividing the world into concepts are significantly different from, say, European conceptual world image. The same can be said about the conceptual world image of people living Far North, as the number of shades of white in it is far beyond traditional European perception. 
After taking into account everything mentioned above we believe that the ultimate definition of concept is provided by V. Maslova who defines concept as "a semantic unit marked with linguistic and cultural particularity which characterizes to some extent the people belonging to certain ethnic culture" [3].

\section{Categorization Issues}

One of the conditions that provide normal flow of conceptualization and categorization processes in human mind is a semiotic system which consolidates and represents the knowledge obtained, as well as the information processing results, in the form of objectified concepts and categories: "Linguistic shapes objectify various knowledge structures which are stored in human's memory as categories" [12]. N. Boldyrev expresses the same idea: "Representation of knowledge in language is the result of two basic cognitive processes performed with use of language - conceptualization and categorization" [13]. Eventually, E. Kubryakova says: "In general we have every reason to affirm that categorization of the world - that is putting concepts into specified groups, classes etc. - into categories, is ultimately of linguistic nature. $\langle\ldots\rangle$ No science, no knowledge area, no theoretical and practical activity are possible without creating terminological systems, without establishing scholarly (linguistic) apparatus to represent them" [1].

In such manner, categorization plays very important role in cognition, apprehension and representation of the world around us. E. Kubryakova notes, "Issues concerning conceptualization and categorization of the world are key issues of cognitive science, later cognitive linguistics" [8]. N. Boldyrev also underlines the significance of categorization as one of the basic cognitive processes: "Many issues that cognitive linguistic faces at the modern stage of its development are connected with study of linguistic methods and mechanisms for representation of knowledge. In this case, special attention is paid to linguistic categorical systems" [10].

V. Zabotkina and E. Boyarskaya go on further on this idea: "Study of the categorization process is important for every linguistic research, as this process is one of the elements the use of language is based on" [14].

A. Levitskiy also stresses out the relevance of studying categorization mechanisms: "Issue of categorization of linguistic units has always been relevant throughout the history of linguistics" [15]. The author also notes, “... human's aspiration for categorization of reality is one of the most important aspects of his mental activity" [15].

While speaking about the importance and relevance of categorization as cognitive process, we should also pay attention to another aspect of the problem. Different people - even within one nation with everybody sharing common linguistic and cultural setup - divide reality into categories in a slightly different fashion.

On the other hand, all people perform their thinking activity in their unique manner; as s result, they build up different individual linguistic world images and use different ways of objectifying apparently same concepts and categories. This feature stands out even more when we compare two different cultures. One of the examples may be difference between English and Russian terms of age periodization.

Keeping this in mind, we should mention the wellknown proposition by F. de Saussure which reads that language is not a nomenclature; there is also a number of statements by modern scientists which lay in the same area of thought. For example, D. Chandler notes, "Language categories are not simply a consequence of some predefined structure of the world ... They are not 'natural' concepts which are simply 'reflected' in language" [16].

V. Demyankov comes up with a similar idea: "By means of language we construct what take for reality, so we can't say that language provides reference to the real world as a really existing independent matter" [2].

Indeed, one of the most significant issues of categorization as cognitive process is the exactly categories (which are non-verbal in the first place) are objectified by means of natural language. Language is inevitably considered as something secondary towards brainwork, irrespectively of how important its functions are in terms of cognitive processes.

Individual approach to dividing the world into concepts and categories can be seen among people belonging to the same linguistic and cultural environment. Furthermore, it can be seen not only in everyday life but also at the professional level. One of the examples is dividing human pelvis as anatomical object into imaginary parts. In fact, pelvis does not have any obvious external marks that allow dividing it precisely and unambiguously into pieces and creating a classification of its parts. As a result, there is a number of various classifications of one and the same anatomical object based on different dividing criteria. For example, a traumatologist and an obstetrician-gynecologist would divide pelvis into completely different imaginary parts, each of them according to the specific nature of his subject field. This may be one of the reasons why there is no united classification of pelvis bones.

\section{Conclusion}

So, we have figured out that cognitive activity follows basically the same patterns, but individual features of brainwork must be taken into account as well. As we have noted above, consciousness of each person is individual and unique; for this reason, all individual conceptual world images are different, as well as methods used by people to objectify similar concepts and categories.

We have also considered some issues of conceptualization and categorization of the world by means of natural language. Some of them are caused by sometimes unclear definition of the 'concept' notion given by various cognitive scientists, as well as different meanings of this notion found in the number of academic disciplines related to cognitive linguistics. Other issues 
are called forth by unobvious nature of objectifying concepts by means of natural language.

Finally, we should not forget that processes of conceptualization and categorization, as well as representation of concepts and categories by means of natural language are influenced by individual mental features. We should also remember about problems caused by linguistic and cultural differences between people.

\section{References}

1. E.S. Kubryakova, Cognitive language research 7, 1519 (2006)

2. V.Z. Demyankov, Cognitive language research 7, 26-44 (2010)

3. V.A. Maslova, Cognitive linguistics (TetraSistems, Minsk, 2008)

4. E.I. Golovanova, Introduction to cognitive science of terminology (Flinta, Moscow, 2011)

5. N.N. Boldyrev, Horizons of modern linguistics, 38$50,(2009)$
6. A.L. Sharandin, Questions of Cognitive Linguistics 34, 75-81 (2013)

7. Z.D. Popova, I.A. Sternin, Cognitive linguistics (AST: Vostok-Zapad, Moscow, 2007)

8. E.S. Kubryakova, Language and knowledge: process of obtaining knowledge about language (Yaziki slavyanskoy kulturi, Moscow, 2004)

9. V.V. Krasnykh, "At home" along "strangers": myth or reality? (Gnozis, Moscow, 2003)

10. N.N. Boldyrev, Cognitive language research 7, 4559 (2010)

11. O.A. Koval, Vestnik MGOU 1, 39-44 (2007)

12. O.N. Babushkina, Questions of Cognitive Linguistics 2, 31-41 (2013)

13. N.N. Boldyrev, Cognitive language research 14, 5260 (2013)

14. V.I. Zabotkina, E.L. Boyarskaya, Cognitive language research 7, 60-68 (2010)

15. A.E. Levitskiy, Cognitive language research 7, 110118 (2010)

16. D. Chandler, Semiotics: the Basics (Routledge, London, 2007) 